\title{
Nonmonotonic Reasoning with Quantified Boolean Constraints
}

\author{
Chris Pollett ${ }^{1}$ and Jeffrey B. Remmel ${ }^{2}$ \\ 1 Department of Mathematics, University of California at San Diego, La Jolla, CA \\ 92903, e-mail: cpollett@math.ucsd.edu \\ 2 Department of Mathematics, University of California at San Diego, La Jolla, CA \\ 92903. Currently with Sagent Corporation, Bellvue, WA, 98004, e-mail: \\ jremmel@sagent.com
}

\begin{abstract}
In this paper, we define and investigate the complexity of several nonmonotonic logics with quantified Boolean formulas as constraints. We give quantified constraint versions of the constraint programming formalism of Marek, Nerode, and Remmel [15] and of the natural extension of their theory to default logic. We also introduce a new formalism which adds constraints to circumscription. We show that standard complexity results for each of these formalisms generalize in the quantified constraint case. Gogic, Kautz, Papadimitriou, and Selman [8] have introduced a new method for measuring the strengths of reasoning formalisms based on succinctness of model representation. We show a natural hierarchy based on this measure exists between our versions of logic programming, circumscription, and default logic. Finally, we discuss some results about the relative succinctness of our reasoning formalisms versus any formalism for which model checking can be done somewhere in the polynomial time hierarchy.
\end{abstract}

\section{Introduction}

The motivation for this paper arose naturally from the theory of constraint programs and constraint models as proposed by Marek, Nerode, and Remmel [15]. That is, Marek, Nerode, and Remmel extended the logic programming with negation formalism to encorporate arbitrary constraints. The constraints considered by Marek, Nerode, and Remmel are not restricted to statements on real numbers as in $\operatorname{CLP}(\mathbf{R})$, see [12]. Instead, they defined a general notion of constraint programs and constraint models and showed that stable models of logic programs as well as the supported models of logic programs are just special cases of constraint models of constraint programs.

In the Marek, Nerode, Remmel theory, the constraint of a clause is not restricted to be of a certain form or even to be expressible in the underlying language of the logic program. A constraint program clause is of the form

$$
p \leftarrow a_{1}, \ldots a_{n}: \Psi
$$

where no particular restrictions were placed on the formula $\Psi$. The formula $\Psi$ is called a constraint formula. The idea is that the constraint formula of a clause 
incorporates the conditions that need to be satisfied by (some) parameters before we start to evaluate the remaining goals in the body of the clause. Thus the constraint controls the applicability of the clause, i.e. , if it fails, then the rule cannot fire. The novel feature of their theory is that the constraints are supposed to be true in the model consisting of the atoms computed in the process. That is, one allows for an a posteriori justification of the rules applied in the process of computation. At first glance this is a different phenomenon than occurs in constraint logic programming. There the constraints are applied a priori. Indeed, in constraint logic programming, once one finds the multidimensional region containing the solutions of the constraint, the subsequent steps can only refine this description in that the solutions must come from that region. The constraints allowed in a constraint program may be very diverse and complex formulas. They may be formulas in the underlying language of the program or they may be formulas in second order or even infinitary logic. An example of this type of constraint is a parity example where the constraint is a statement about the parity of the (finite) putative model of the program. This type of the constraint is a formula of infinitary logic if the Herbrand base is infinite. The only requirement for a constraint is that there is a method to check if the constraint holds relative to a possible model.

The motivation for allowing more general constraints originally came from the certain applications in control theory of real-time systems. The basic idea is that we sample the plant state at discrete intervals of time, $\Delta, 2 \Delta, \ldots$ Based on the plant measurements, a logic program will compute the control to be used by the plant for the next $\Delta$ seconds so that the plant state will be guaranteed to meet certain required specifications. One possible way for such logic programs to operate is that the set of rules with which we compute at any give time $n \Delta$ is a function of the observations of the state of the plant at time $n \Delta$. In this fashion, we can view the plant state at time $n \Delta$ as determining which constraints of the rules of the logic program are satisfied and hence which rules can fire at time $n \Delta$. In such a situation, we cannot expect that we will have the constraints which are necessarily in the same language as the underlying language of the program. For example, $\Psi$ could be a formula that asserts that a particular integral has value one. Therefore it seems necessary to step out of the current constraint logic programming paradigm. Another application of the same type is to have a logic program controlling inventory via a deductive database. In this case, we may want to change the rules of the deductive database as a function of outside demand. Once again one could view the satisfaction of the constraints as depending on the database for the inventory and the set of orders that come in at a given time. In this way one can vary the set of applicable rules as function of the current inventory and demand.

The constraint formulas control the applicability of a clause during program evaluation. They allow for an increased flexibility in the forms of data that can be handled by logic programs. The actual evaluation of these constraint formulas might be handled by specialized hardware and so model search for these programs is potentially feasible. Nevertheless, the evaluation of constraint models 
which are the natural extension of the stable logic semantics to programs built out of such clauses requires checking these constraints. Hence the checking of constraints becomes a new source of complexity in the the evaluation of constraint models. Thus to study the complexity of this type logic programming with generalized constraints, or indeed any form of nonmonotonic logic with generalized constraints, it is useful to develop constraint theories of various complexity.

Quantified propositional formulas provide a natural tool for developing constraint theories of varying complexity. This is because restricting the quantifier depth of such formulas yields natural complete problems for various levels of the polynomial hierarchy. From the practical point of view quantified Boolean formulas as constraints allow one to check global properties of a database or plant in a way which would be much more cumbersome if one had to rely on propositional constraints alone.

In this paper, we define and investigate the complexity of several nonmonotonic logics with quantified Boolean formulas as constraints. In particular, we give quantified constraint versions of constraint programming of Marek, Nerode, and Remmel [15] and the natural extension of their theory to default logic. We also introduce a new formalism which adds constraints to circumscription. We show that standard complexity results $[2,14]$ for each of these formalisms generalize in the quantified constraint case. If our constraint formulas are restricted to be $Q B F_{k}$, quantified Boolean formulas with $k$ alternations of quantifiers, then model existence problem in constraint programming $\left(L P_{k}\right)$ is $\Sigma_{k+1}^{p}$-complete. Similarly, the model existence problem for our version of default logic $\left(D L_{k}\right)$ is $\Sigma_{k+2}^{p}$-complete. For our version circumscription $\left(C C_{k}\right)$ we show that it is $\Pi_{k+2^{-}}^{p}$ complete to determine if a given variable is in all models of a circumscribed program. The fact that completeness results for these theories lift so uniformly up through the polynomial hierarchy, i.e. our version of logic programming always remains one level below our versions of circumscription and default logic, illustrates the crucial role that constraints play in complexity results for nonmonotonic theories.

A new method for comparing the relative strength of reasoning formalisms was developed by Gogic, Kautz, Papadimitriou, and Selman [8] (see also Papadimitriou [17] and Gogic [9]). It is based on the succinctness with which a formalism can express a particular collection of models. The idea is that given any reasoning formalisms $A$ and $B$, we say that $A$ is at least as representationally succinct as $B$, written $B \leq_{s} A$, if for each knowledge base $\phi_{B}$ in the reasoning formalism $B$, there is a knowledge base $\phi_{A}$ in the reasoning formalism $A$, whose size is polynomial bounded in the size of $\phi_{B}$ such that $\phi_{B}$ and $\phi_{A}$ are defined over the same free variables and have the same set of models. Note that there is no requirement that $\phi_{A}$ be effectively computed from $\phi_{B}$. It is easy to see succinctness criteria induce a transitive, reflexive relation $\leq_{s}$ on the class of reasoning formalisms. Gogic, Kautz, Papadimitriou, and Selman showed that there is a rather remarkable hierarchy that results among various reasoning formalisms such as propositional logic, horn logic, circumscription, and default logic given that the polynomial time hierarchy does not collapse at low levels. 
We shall show that there is a natural hierarchy that exists among the theories $L P_{k}, D L_{k}$, and $C C_{k}$ introduced in this paper. We show that $L P_{k}<_{s} D L_{k}$ and $L P_{k}^{*}<_{s} C C_{k}<_{s} D L_{k}$. The strictness of these inclusions is under the assumption that the polynomial hierarchy does not collapse. $L P_{k}^{s u p}$ is $L P_{k}$ where we consider supported models of programs rather than stable ones. $L P_{k}^{*}$ is $L P_{k}$ restricted to programs whose supported models are pairwise incomparable. With a slightly weaker notion of succinctness $\leq_{w s}$ where one does not insist that $\phi_{A}$ and $\phi_{B}$ have the same set of variable, one can show:

$$
L P_{k}^{*} \equiv{ }_{w s} L P_{k}<_{w s} C C_{k} \leq_{w s} D L_{k} \equiv{ }_{w s} L P_{k+1} .
$$

We also show that $L P_{k} \leq_{w s} L P_{k}^{s u p}$ and $L P_{k} \leq_{s} L P_{k}^{s u p}$. Thus $L P_{k} \equiv L P_{k}^{s u p}$. Similar results are proven for default logic and circumscription.

The results of this paper are presented in four sections. In the first three sections, we develop our versions of logic programming, circumscription, and default logic. In the fourth section we discuss our succinctness results. Besides the above set of inclusions, in the fourth section, we discuss some results about the relative succinctness of our reasoning formalisms versus any formalism for which model checking can be done somewhere in the polynomial time hierarchy.

Finally, we wish to thank Victor Marek for carefully reading our first draft to the paper and for many suggested improvements.

\section{Logic Programming}

We will be considering clauses of the form

$$
p \leftarrow a_{1}, \ldots, a_{n}: B_{1}\left(\boldsymbol{b}_{1}\right), \ldots, B_{n}\left(\boldsymbol{b}_{m}\right)
$$

where $p, a_{1}, \ldots, a_{n}$ are propositional variables and $B$ is a quantified Boolean formula[16]. For each $i$, the $\boldsymbol{b}_{i}$ represents the free propositional variables in $B_{i}$. We call $p$ the conclusion of the clause, we call the $a_{i}$ 's the premises of the clause, and we call the $B_{i}$ 's constraint formulas.

A logic program with quantified Boolean constraints is a collection of clauses of the above type. We will often call this just a constraint logic program or program. The Herbrand Universe for such a program consists of all propositional variables appearing free (unquantified) somewhere in the program. Let $P$ be a program. Let $M$ be a subset of the Herbrand Universe of $P$ and let $\nu_{M}$ be the truth assignment such that $\nu_{M}(x)=1$ if and only if $x \in M$. We denote by $P_{M}$ the logic program obtained from $P$ by deleting those clauses whose constraints are not satisfied by $\nu_{M}$ and by deleting the constraints from the remaining clauses. $P_{M}$ is a Horn program and has a least model $N_{M}$. We say that $M$ is a stable model of $P$ if it is the case that $M=N_{M}$.

Every truth assignment $\nu$ uniquely extends to a truth assignment $\bar{\nu}$ which evaluates all the quantified Boolean formulas and satisfies the usual Tarskian conditions for satisfaction. A supported model for a logic program is a truth assignment $\nu$ to its Herbrand Universe such that for each variable p, $\nu(p)=1$ 
if and only if there is a clause of type $(*)$ such that $\nu\left(a_{1}\right)=1, \ldots \nu\left(a_{n}\right)=1$, and $\bar{\nu}\left(B_{1}\left(\boldsymbol{b}_{\mathbf{1}}\right)\right)=1, \ldots \bar{\nu}\left(B_{m}\left(\boldsymbol{b}_{\boldsymbol{m}}\right)\right)=1$. Otherwise, $\nu(p)=0$. The clause $p \leftarrow$ : can be used to force $\nu(p)$ to be 1 . We say that $a$ is in a model $\nu$ if $\nu(a)=1$. If $\nu(a)=0$ then $a$ is out of $\nu$. Every stable model of $P$ is a supported one, but the reverse need not be true. We will use supported models when we prove our succinctness results. Unless we state otherwise, however, when we say model of a logic program we mean stable model.

We write $\Sigma_{k}^{q}$ to denote the set of quantified Boolean formulas with at most $k$ alternations of quantifier type and whose outermost quantifier is an $\exists$. Similarly, we write $\Pi_{k}^{q}$ denote the set of quantified Boolean formulas with at most $k$ alternations of quantifier type and whose outermost quantifier is an $\forall$. In both cases, unless we say we are dealing with only sentences, we assume our formulas have free variables. Lastly, we write $Q B F_{k}$ to denote Boolean combinations of these two classes. In the $k=0$ case all of the above classes are the same: The class of propositional formulas. We recall that the problem of determining whether a $\Sigma_{k}^{q}$-sentence is true is $\Sigma_{k}^{p}$-complete and the problem of determining whether a $\Pi_{k}^{q}$-sentence is true is $\Pi_{k}^{p}$-complete. Given an assignment $\nu$ to the free variables of a $Q B F_{k}$ formula $B$, the problem of determining whether or not $\bar{\nu}(B)$ is true is in $\Delta_{k+1}^{p}$.

Definition 1. $L P_{k}$ is the class of finite logic programs whose constraints are all in $Q B F_{k} . L P_{\infty}=\cup_{k \geq 0} L P_{k}$.

Let us pause a moment here to compare the class $L P_{0}$ to logic programs that allow for yet simpler constraints. Some results on such programs were proved by Marek, Nerode, and Remmel in [15]. Specifically, for programs with clauses that have propositional constraints they construct an equivalent program in the same language where all the constraints are conjunctions of literals. The reason why this is possible is that constraints distribute with respect to disjunction. That is, a program transformation where replace a clause $p \leftarrow a_{1}, \ldots a_{n}: B_{1} \vee B_{2}$ by two clauses, $p \leftarrow a_{1}, \ldots a_{n}: B_{1}$ and $p \leftarrow a_{1}, \ldots a_{n}: B_{2}$, does not change semantics. Consequently, all we need to do is to put constraints in disjunctive normal form (disjunction of conjunctions) and then distribute. We repeat it for all clauses of $P$ if necessary.

This conversion yields a program potentially exponential in the size of the original program. If however one allows for additional variables then we can find a polynomial transformation. We will outline it below. First, by taking the conjunction of all constraints, we can assume that each clause has just one constraint. Indeed, each of the constraints of a clause is satisfied if and only if their conjunction is satisfied. Next, since we do not changes the set of stables of a program if we replace any constraint in the program by a logically equivalent constraint, we can eliminate implications and push the negations downwards to the level of literals. Thus we can assume that the constraints are built out of literals by means of conjunctions and alternatives. Since the constraints split with respect to alternatives, all we need to do is to handle conjunctions. We do 
this as follows. Given a clause of the form

$$
C=p \leftarrow a_{1}, \ldots, a_{m}: \mathbb{A}_{j=0}^{r} B_{j}
$$

we introduce $r$ new constants $b_{1}, \ldots, b_{r}$, eliminate $C$ and put into $P$

$$
\begin{aligned}
& p \leftarrow a_{1}, \ldots, a_{m}, b_{1}, \ldots, b_{n}: \\
& b_{1} \leftarrow: B_{1} \\
& \ldots \\
& b_{n} \leftarrow: B_{n}
\end{aligned}
$$

After the execution of these operations, the resulting program $P^{*}$ (clearly polynomial in size of $P$ ) has the following properties. First, every stable (constraint) model of $P$ uniquely extends to a model of $P^{*}$. Second, all the constraints of clauses of $P^{*}$ are either tautologies, or a single literals. We notice that in the context of general logic programs this result is well-known. In the terminology of Section 5 we proved that for the class $L P^{*}$ of programs with a single constraint which is a tautology or a literal, we have $L P_{0} \leq_{w s} L P^{*}$ (and consequently $L P_{0} \equiv{ }_{w s} L P^{*}$ as well).

\section{Theorem 1.}

1. The problem $\exists C M L P_{k}$ of determining whether an $L P_{k}$ program has a model is $\Sigma_{k+1}^{p}$-complete.

2. The problem $\exists C M L P_{\infty}$ of determining whether a finite $L P_{\infty}$ program has a model is PSPACE-complete.

Proof. First, we show $\exists C M L P_{k} \in \Sigma_{k+1}^{p}$. Let $P \in L P_{k}$. We guess a model for $P$ then check which of $P$ 's clauses have their constraint formulas satisfied. As we said above, given a truth assignment, checking a $Q B F_{k}$ formula can be done in $\Delta_{k+1}^{p}$. We delete the clauses whose constraint formulas are not satisfied. The remaining clauses have their constraint formulas satisfied so we can delete the constraint formulas for these formulas. The resulting program is a Horn program and finding a minimal model for a Horn program can be done in linear time $[3,14]$. Thus, this whole algorithm can be done in $N P\left(\Delta_{k+1}^{p}\right)$. Therefore, $\exists C M L P_{k} \in \Sigma_{k+1}^{p}$.

For completeness, let $B\left(b_{1}, \ldots, b_{m}\right) \in Q B F_{k}$. Consider the following logic program $P^{*}$. It has four groups of clauses:

$$
\begin{array}{ll}
b_{i} \leftarrow: \neg \bar{b}_{i} & i=1, \ldots, m \\
\overline{b_{i}}: \neg \neg b_{i} & \\
\gamma \leftarrow b_{i}, \bar{b}_{i}: & i=1, \ldots, m \\
b_{i} \leftarrow: \neg \gamma & \\
\bar{b}_{i} \leftarrow: \neg \gamma & \\
\gamma \leftarrow: B\left(b_{1}, \ldots b_{m}\right) &
\end{array}
$$

Because of clauses (2) and (3), $P^{*}$ has a model $M$ only if $\gamma \in M$. However $\gamma$ can not be derived by a clause of type (2) since clauses of type (3) only fire if we 
do not have $\gamma$ and clauses of type (1) will only derive one of $b_{i}$ or $\bar{b}_{i}$. Hence, $\gamma$ must be derived by (4). Thus, to derive $\gamma$ we must be able to solve the problem of whether $\exists \boldsymbol{b} B(\boldsymbol{b})$ is valid. But determining whether a $\Sigma_{k+1}^{q}$ formula is valid is a $\Sigma_{k+1}^{p}$-complete problem. Thus $\exists C M L P_{k}$ is $\Sigma_{k+1}^{p}$-complete.

The $L P_{\infty}$ case is proven in a similar fashion.

\section{Theorem 2.}

1. The problem of deciding whether a given variable a is in a model of an $L P_{k}$ program is $\Sigma_{k+1}^{p}$-complete.

2. The analogous problem for $L P_{\infty}$ it is PSPACE-complete.

Proof. The problem is in $\Sigma_{k+1}^{p}$ since we can just guess a truth assignment $\nu$ such that $\nu(a)=1$ and check that it is a model. To see that our problem is $\Sigma_{k+1}^{p}$-complete notice that the problem of whether $\gamma$ is in an extension of $P$ in Theorem 1 is equivalent to whether a $\Sigma_{k+1}^{q}$-formula is valid.

The $L P_{\infty}$ case is similar.

Remark 1. It is not hard to generalize the notion of logic programming with quantified Boolean constraints: One needs only generalize the notion of quantified Boolean formula. For instance, rather than take our atoms of quantified Boolean formulas to be just propositional variables we could let them be propositional variables and expressions of the form $a_{1} a_{2} \ldots a_{n} \in A$. That is, checking if the concatenation of some string propositional variables is in an oracle $A$. Given an assignment $\nu$, we define $\bar{\nu}\left(a_{1} a_{2} \ldots a_{n} \in A\right)=1$ if and only if the string $\nu\left(a_{1}\right) \nu\left(a_{2}\right) \ldots \nu\left(a_{n}\right)$ is in the set $A$. Thus, there is a well defined semantics for such formulas. So we can define the classes $\Sigma_{k}^{q}(A), \Pi_{k}^{q}(A)$, and $Q B F_{k}(A)$ and use them in our logic programming theories. Hence, we can define $L P_{k}(A)$ to be finite logic programs with $Q B F_{k}(A)$ constraints. From Goldsmith Joseph [10] and Schöning [18] it is known that $S A T_{k}(A)$ is $\Sigma_{k+1}^{p}(A)$-complete. Hence, using exactly the same arguments we can generalize the theorems we have just obtained for $L P_{k}$.

\section{Theorem 3.}

1. The problem of deciding whether an $L P_{k}(A)$ program has a model is $\Sigma_{k+1}^{p}(A)$ complete.

2. The analogous problem for $L P_{\infty}(A)$ it is PSPACE $(A)$-complete.

\section{Theorem 4.}

1. The problem of deciding whether a given variable a is in a model of an $L P_{k}(A)$ program is $\Sigma_{k+1}^{p}$-complete.

2. The analogous problem for $L P_{\infty}(A)$ it is PSPACE $(A)$-complete.

\section{Circumscription}

There are several ways one could generalize circumscription to higher levels of the polynomial hierarchy. Perhaps the easiest way would be to take $Q B F_{k}$ formulas 
and circumscribe them directly. The circumscribed models of a $Q B F_{k}$ formula $B(\boldsymbol{a})$ would be the minimal models of $B$ under set-theoretic inclusion.

However, in this paper we are taking the approach that one should separate constraints, which might be possible to calculate a priori or possibly on some special device, from the computational part of the reasoning scheme. Hence, our version of constraint circumscription will consist of circumscribed programs which are sequences of clauses of the form $B(\boldsymbol{a}) \leftarrow: C(\boldsymbol{b})$, where $B$ is a propositional formula and $C$ is a $Q B F_{k}$ formula. In such a clause, $B$ is called the relational formula and $C$ is called the constraint formula. As usual the Herbrand Universe for such a program will consist of all the free variables which appear in the program. Given a circumscribed program $P$ and a subset $S$ of the Herbrand Universe $U$, let $\nu_{S}$ be the variable assignment which makes element of $S$ true and those of $U \backslash S$ false. Then we define $P_{S}$ to be the following set of relational formulas:

$$
\left\{B \mid B \leftarrow: C \in P \wedge \bar{\nu}_{S}(C)=1\right\} .
$$

We use $\mathbb{A} P_{S}$ to denote the conjunction of formulas in $P_{S}$. Given a circumscribed program $P$ and a set of variables $M$ we define $C I R C[P ; M]$ to be the secondorder formula:

$$
\bigwedge P_{M} \wedge \neg \exists m\left[\wedge P_{m} \wedge m \subset M\right] \text {. }
$$

A model for a circumscribed program $P$ will be a variable assignment $\nu_{M}$ such that $C I R C[P ; M]$ is true.

Definition 2. $C C_{k}$ is the class of all finite constraint circumscription programs with $Q B F_{k}$ constraints. $C C_{\infty}=\cup C C_{k}$.

In the simplest common form of (propositional) circumscription one considers a single propositional formula $F$ and looks for minimal models of $F$ with respect to set theoretic inclusion. These minimal models are called the models of the circumscribed formula $F$. It is not hard too see that given a propositional formula $F$, the $C C_{0}$ program $F \leftarrow$ : has the same models as the circumscribed formula $F$. Given a $C C_{0}$ program with clauses

$$
C\left(\boldsymbol{a}_{\boldsymbol{i}}\right) \leftarrow: D\left(\boldsymbol{a}_{\boldsymbol{i}}\right) \text { for } 1 \leq i \leq n
$$

it is also not hard to see that this program has the same models as the circumscribed formula

$$
\mathbb{M}_{i} D\left(\boldsymbol{a}_{\boldsymbol{i}}\right) \supset C\left(\boldsymbol{a}_{\boldsymbol{i}}\right) .
$$

Thus, the theory $C C_{0}$ coincides with the simplest common form of circumscription.

Our next result is a generalization of a result of Eiter and Gottlob [4].

\section{Theorem 5.}

1. The problem $\forall C C M_{k}$ of determining whether a given variable is in every model of a $C C_{k}$ program is $\Pi_{k+2}^{p}$-complete.

2. The analogous problem for $\forall C C M_{\infty}$ is PSPACE-complete. 
Proof. Let $P$ be a $C C_{k}$ program. To check if $x$ is in all models (recall that a model here is a model of circumscribed formula) of $P$ we universally examine truth assignments $\nu_{M}$. We then check if there exists a smaller truth assignment, $\nu_{m}$, such that either $\bar{\nu}_{M}\left(\mathbb{X} P_{M}\right)=0$ or $\bar{\nu}_{m}\left(\mathbb{X} P_{m}\right)=1$ or $\nu_{m}(x)=1$. If there exists $M$ and $m$ for which this is not the case, then $x$ is not in all models. Hence, $\forall C C M_{k}$ is in $\Pi_{k+2}^{p}$.

To see $\forall C C M_{k}$ is $\Pi_{k+2}^{p}$-complete let $F$ be a $\Pi_{k+2}^{q}$ formula $\forall \boldsymbol{x} \exists \boldsymbol{y} E(\boldsymbol{x}, \boldsymbol{y})$ where $E \in \Pi_{k}^{q}$. Let $\boldsymbol{z}, u$, and $w$ be new variables. Consider the following $C C_{k}$ program:

$$
\begin{aligned}
& \mathbb{X}_{i}\left(x_{i} \not \equiv z_{i}\right) \leftarrow: \\
& u \leftarrow: E(\boldsymbol{x}, \boldsymbol{y}) \\
& \neg u \leftarrow: \neg E(\boldsymbol{x}, \boldsymbol{y}) \\
& {\left[u \vee\left(w \wedge \mathbb{A}_{j} y_{j}\right) \leftarrow \leftarrow:\right.}
\end{aligned}
$$

We claim $F$ is true if and only $u$ is in every model of $P$. Clause (1) ensures that if $m_{1} \subseteq m_{2}$ are models, then $m_{1}$ must agree with $m_{2}$ on the $x_{i}$ 's and $z_{i}$ 's and guarantees for each possible subset of the $x_{i}$ 's there is a model of $P$ with exactly those $x_{i}$ 's. Suppose $u$ is in every model of $P$. We want to show $F$ is true. Let us assume $F$ is false and derive a contradiction. $F$ being false implies $\exists \boldsymbol{x} \forall \boldsymbol{y} \neg E(\boldsymbol{x}, \boldsymbol{y})$. Let $\boldsymbol{x}$ be such that $\forall \boldsymbol{y} \neg E(\boldsymbol{x}, \boldsymbol{y})$. Let $M$ be the model containing $\boldsymbol{x}$. Then by clause (3), \\ $P_{M}$ must have $\neg u$ as a conjunct. So $u$ will not be in this minimal model. This contradicts the assumption that $u$ was in every model.

For the other direction, suppose $F$ is true. We want to show every model of $P$ contains $u$. Let $M$ be a model of $P$. Suppose $\nu_{M}(E(\boldsymbol{x}, \boldsymbol{y}))=0$. Then $\mathbb{\wedge} P_{M}$ would be:

$$
\mathbb{A}_{i}\left(x_{i} \not \equiv z_{i}\right) \wedge \neg u \wedge\left[u \vee\left(w \wedge \mathbb{X}_{j} y_{j}\right)\right]
$$

So $w$ and all the $y_{j}$ s would have to be in $M$. But $F$ being true means we could have found a smaller model since there exists a choice of $y_{i}$ s making $E$ true and hence we can make $w$ false in that model. Hence, $M$ must have $\nu_{M}(E(\boldsymbol{x}, \boldsymbol{y}))=1$. But then by (2), $M$ must contain $u$.

This theorem generalizes when one adds oracle sets to the theories. Hence, the corresponding problem for $C C_{k}(A)$ is $\Pi_{k+2}^{p}(A)$-complete.

\section{Default Logic}

We now develop a version of default logic similar in spirit with the versions of logic programming and circumscription we have just developed.

A quantified Boolean default theory, or just default theory, is a pair $\langle D, W\rangle$ where $D$ is a collection of default rules:

$$
\frac{\alpha: B_{1}\left(\boldsymbol{b}_{\mathbf{1}}\right), \ldots, B_{m}\left(\boldsymbol{b}_{\boldsymbol{m}}\right)}{\gamma}
$$

and $W$ is a set of propositional formulas. The $\alpha$ and $\gamma$ in each rule are propositional formulas and the $B_{i}$ are quantified Boolean formulas with free variables 
$\boldsymbol{b}_{\boldsymbol{i}} . \alpha$ is called the prerequisite, the $B_{i}$ are called the constraints, and $\gamma$ is called the consequent of the rule. The Herbrand base, $U$ for a default theory is the set of free variables appearing in the default rules and $W . C n(W)$ is the set of all formulas provable from $W$ using propositional tautologies and modus ponens. Given a set $S$ of propositional formulas, a default rule is said to be $S$-applicable if $B_{i} \cup S$ is consistent for each justification $B_{i}$. If the prerequisite of a default rule also happens to be in $S$, we say that the rule is strongly $S$-applicable. We write $D_{S}$ for the set or rules

$$
\left\{\frac{\alpha}{\gamma} \mid \frac{\alpha: B_{1}\left(\boldsymbol{b}_{\mathbf{1}}\right), \ldots, B_{m}\left(\boldsymbol{b}_{\boldsymbol{m}}\right)}{\gamma} \in D \text { an } S \text {-applicable rule }\right\} .
$$

$D_{S, w}$ is the subset of $D_{S}$ generated by strongly $S$-applicable rules. Given a set of rules $X, C n^{X}(W)$ is the set of all formula which can be proved from $W$ using propositional tautologies, modus ponens, or rules from $X$.

An extension for a default theory $\langle D, W\rangle$ is a set of formulas $S$ such that $C n^{D_{S}}(W)=S$. A stable model for a default theory $\langle D, W\rangle$ is a truth assignment satisfying an extension of $\langle D, W\rangle$. A weak extension for a default theory $\langle D, W\rangle$ is a set of formulas $S$ such that $W \subseteq S$ and $C n^{D_{S, w}}(S)=S$. A supported model for a default theory $\langle D, W\rangle$ is a satisfying truth assignment to the variables of a weak extension of $\langle D, W\rangle$. It should be noted that an inconsistent theory cannot have either type of model. When we say model of a default theory we mean stable model. Supported models of default theories will be discussed again in the succinctness section.

We define $B^{D_{S}}(T)=T \cup\left\{\gamma \mid \frac{\alpha}{\gamma} \in D_{S}\right.$ and $\left.\alpha \in C n(T)\right\}$. To denote iterating this operation $n$ times we write $B^{D_{S}} \uparrow n(T)$, and we write $B^{D_{S}} \uparrow \omega(T)$ to denote iterating this operation $\omega$ times. If $\langle D, W\rangle$ is a finite theory than there exists and $n$ such that $B^{D_{S}} \uparrow n(W)=B^{D_{S}} \uparrow(n+1)(W)=B^{D_{S}} \uparrow \omega(W)$.

We begin with a couple of observations about default theories with quantified Boolean constraints. Their proofs are the same as in the classical default theory case.

1. A theory $S$ is an extension for a default theory $\langle D, W\rangle$ if and only if $S=$ $C n\left(B^{D S} \uparrow \omega(W)\right)$.

2. If $S$ is an extension of $\langle D, W\rangle$ then there is a subset $Z$ of the consequences of $D$ such that $S=C n(W \cup Z)$.

Definition 3. $D L_{k}$ is the class of all finite default theories all of whose rules have $Q B F_{k}$ constraints. $D L_{\infty}=\cup D L_{k}$.

Notice $D L_{0}$ is exactly the same as the usual default logic.

We begin with a generalization of the fundamental result of Gottlob [11].

\section{Theorem 6.}

1. The problem $\exists E D L_{k}$ of deciding whether $\langle D, W\rangle \in D L_{k}$ has an extension is $\Sigma_{k+2}^{p}$-complete.

2. The analogous problem for $D L_{\infty}$ it is PSPACE-complete. 
Proof. Let $\langle D, W\rangle \in D L_{k}$. To show the problem of finding an extension is in $\Sigma_{k+2}^{p}$ we use the two observations above. We guess a subset $Z$ of the conclusions of the rules. We take $S$ to be $C n(W \cup Z)$ and check that $S=C n\left(B^{D_{S}} \uparrow\right.$ $\omega(W))$. First we must figure out what $D_{S}$ is. It is not hard to see that a default $d=\frac{\phi: B_{1}\left(\boldsymbol{b}_{1}\right), \ldots B_{m}\left(\boldsymbol{b}_{m}\right)}{\gamma}$ contributes $\frac{\phi}{\gamma}$ if and only if for each $i,\left\{B_{i}\left(\boldsymbol{b}_{\boldsymbol{i}}\right)\right\} \cup W \cup Z$ is consistent. Next, we check if $\left\{B_{i}\left(\boldsymbol{b}_{\boldsymbol{i}}\right)\right\} \cup W \cup Z$ is consistent. This requires looking for a vector $\boldsymbol{b}_{\boldsymbol{i}}$ such that a $\Pi_{k}^{p}$ property holds. Thus, we would need a single $\Sigma_{k+1}^{p}$ oracle call. To do this for each default we would need less than $|D|$ many $\Sigma_{k+1}^{p}$ oracle calls. Next we construct $B=B^{D_{S}} \uparrow \omega(W)$. This takes at most $\left|D_{S}\right|^{2}$ many co-NP calls to check if a given prerequisite is a consequence of the set generated so far. Lastly we need to check that $C n(B)=C n(W \cup Z)$.

Let $\Phi$ be the conjunction of formulas in $B$ and $\Psi$ be the conjunction of formulas in $W \cup Z$. Then checking the above condition amounts to checking if $\Phi \equiv \Psi$ is a tautology. So after our initial guess of $Z$, all our oracle calls are to sets contained in $\Sigma_{k+1}^{p}$. Thus, the entire procedure outlined above is $N P^{\Sigma_{k+1}^{p}}=\Sigma_{k+2}^{p}$.

To see $\exists E D L_{k}$ is $\Sigma_{k+2}^{p}$-complete, let $B(\boldsymbol{a}, \boldsymbol{b}) \in \Sigma_{k}^{q}$ where $\boldsymbol{a}=\left(a_{1}, \ldots, a_{m}\right)$ and $\boldsymbol{b}=\left(b_{1}, \ldots, b_{n}\right)$ are the free variables in $B$. Consider the following default theory $\left\langle D^{*}, W^{*}\right\rangle$ :

$$
\begin{array}{llll}
\text { (1) } & \frac{: a_{i}}{a_{i}} & \frac{\neg a_{i}}{\neg a_{i}} & i=1, \ldots, m \\
\text { (2) } & \frac{: \neg \gamma}{a_{i}} & \frac{: \neg \gamma}{\neg a_{i}} & i=1, \ldots, m \\
\text { (3) } & \frac{: B(\boldsymbol{a}, \boldsymbol{b})}{\gamma} &
\end{array}
$$

$\left\langle D^{*}, W^{*}\right\rangle$ has an extension $S$ implies

$$
C n\left( \pm a_{1} \wedge \pm a_{2} \wedge \ldots \pm a_{m} \wedge \pm \gamma\right) \mid=\gamma
$$

The $\pm a_{i}$ is supposed to be either $a_{i}$ or $\neg a_{i}$ depending upon which of these literals belongs to $S$. From the above we thus have:

$$
C n\left( \pm a_{1} \wedge \pm a_{2} \wedge \ldots \pm a_{m}\right) \models B(\boldsymbol{a}, \boldsymbol{b}) .
$$

Consequently, we have

$$
C n\left( \pm a_{1} \wedge \pm a_{2} \wedge \ldots \pm a_{m}\right) \models \forall \boldsymbol{w} B(\boldsymbol{a}, \boldsymbol{w}) .
$$

This in turn implies that the sentence $\exists \boldsymbol{x} \forall \boldsymbol{w} B(\boldsymbol{x}, \boldsymbol{w})$ is true. We can reverse all the above implications. That is, if $\neg \exists \boldsymbol{x} \forall \boldsymbol{w} B(\boldsymbol{x}, \boldsymbol{w})$, then there is no choice of $\pm x_{i}$ 's which satisfies $\forall \boldsymbol{w} B(\boldsymbol{a}, \boldsymbol{w})$. So $\gamma$ cannot be derived. Thus $\langle D, W\rangle$ has no extensions.

So existence of an extension of default theories of the form $\left\langle D^{*}, W^{*}\right\rangle$ is equivalent to the $\Sigma_{k+2}^{p}$-complete problem of whether a $\Sigma_{k+2}^{q}$ sentence is valid.

$D L_{\infty}$ case is proven in a similar fashion. 


\section{Corollary 1.}

1. The problem of deciding whether $\langle D, W\rangle \in D L_{k}$ has a model is $\Sigma_{k+2}^{p}$-complete.

2. The analogous problem for $D L_{\infty}$ it is PSPACE-complete.

Proof. To see the problem is in $\Sigma_{k+2}^{p}$ let $\langle D, W\rangle \in D L_{k}$. We now slightly modify the procedure to find an extension of a default theory used in Theorem 6 . At the same time, as we guess a subset $Z$ of the conclusion we also guess a model $M$ for $\langle D, W\rangle$. Then when we check that whether $\Phi \equiv \Psi$ is a tautology we also check if $M \models \Phi$. Only if both of these conditions are true is $M$ a model $\langle D, W\rangle$. This procedure is still in $\Sigma_{k+2}^{p}$.

To see that our problem is $\Sigma_{k+2}^{p}$-complete notice the default theory used in Theorem 6 has models if and only if the sentence $\exists \boldsymbol{x} \forall \boldsymbol{w} B(\boldsymbol{x}, \boldsymbol{w})$ is true.

$D L_{\infty}$ case is proven in a similar fashion.

\section{Corollary 2.}

1. The problem of given a formula $\phi$ and deciding whether or not it is in an extension of $\langle D, W\rangle \in D L_{k}$ is $\Sigma_{k+2}^{p}$-complete.

2. The analogous problem for $D L_{\infty}$ it is PSPACE-complete.

Proof. The problem is in $\Sigma_{k+2}^{p}$ since we can use the previous corollary to guess a model $M$ and then check if $M \models \phi$. To see that it is $\Sigma_{k+2}^{p}$-complete notice that the problem of whether $\gamma$ is in an extension of $\left\langle D^{*}, W^{*}\right\rangle$ in Theorem 6 is equivalent to whether a $\Sigma_{k+2}^{q}$-formula is satisfiable.

$D L_{\infty}$ case is proven in a similar fashion.

The theorem and the corollaries above generalize when one adds oracle sets to the theories $D L_{k}$. Hence, the corresponding problems for $D L_{k}(A)$ are $\Sigma_{k+2}^{p}(A)$ complete.

\section{Succinctness}

In [8] a notion of representation succinctness was developed as a means of comparing the relative strength of various reasoning formalisms. We will now establish several succinctness results about nonmonotonic logics with quantified Boolean constraints. These results generalize those of [8].

Definition 4. Let $A$ and $B$ be any reasoning formalisms. We say that $A$ is at least as representationally succinct as $B$, written $B \leq_{s} A$ if the following is true: For each knowledge base $\phi_{B}$ in $B$ there is a knowledge base $\phi_{A}$ in $A$ such that (a) $\phi_{B}$ and $\phi_{A}$ are defined over the same free variables and have the same set of models, and (b) the size of $\phi_{A}$ is polynomially bounded in the size of $\phi_{b}$. We write $A \not_{s} B$ if (a) and (b) fail to hold.

It should be noted that the method of going from $\phi_{A}$ to $\phi_{B}$ need not to be effective. Next, the definition makes it is obvious that $\leq_{s}$ is transitive and reflexive. We can slightly weaken the definition of succinctness by replacing (a) in the above with the following: 
$\left(a^{\prime}\right) \phi_{A}$ contains all of $\phi_{B}$ 's variables and all models of $\phi_{A}$ are expansions of models of $\phi_{B}$.

We will call succinctness with respect to $\left(a^{\prime}\right)$ weak succinctness and will write $B \leq{ }_{w s} A$ if $A$ is at least as weakly representationally succinct as $B$.

It is follows from the definitions that if $B \leq_{s} A$ then $B \leq_{w s} A$. Moreover, $\left(a^{\prime}\right)$ implies that $\phi_{A}$ can not have a model unless $\phi_{B}$ has one.

We adopt the convention that $(k \geq 0)$. Let $L P_{k}$ with respect to supported models will be denoted $L P_{k}^{\text {sup }}$. $L P_{k}$ with respect to stable models we will just write as $L P_{k}$. We then have:

Theorem 7. $L P_{k}^{\text {sup }} \leq_{s} L P_{k}$ and $L P_{k} \leq_{w s} L P^{s u p}$. Thus, $L P_{k} \equiv_{w s} L P_{k}^{\text {sup }}$.

Proof. Let $P$ be an $L P_{k}^{s u p}$ program. A variable $a$ is in a supported model $M$ of $P$ if and only if there is a clause of the form

$$
a \leftarrow: p_{1}, \ldots, p_{n}: B_{1}, \ldots, B_{m}
$$

and $\nu_{M}\left(p_{i}\right)=1$ for $1 \leq i \leq n$ and $\bar{\nu}\left(B_{j}\right)=1$ for $1 \leq j \leq m$. Thus, we can view the premises and constraints of such a clause as a single constraint: namely, $\mathbb{A}_{i} p_{i} \wedge \mathbb{A}_{j} B_{j}$. The program $P^{\prime}$ one obtains by replacing the premises and constraints of clauses in $P$ by such single constraints will still have the same supported models. But now given a set $M, P_{M}^{\prime}$ will consist of clauses of the form $a \leftarrow$. So if $M$ is a stable model any such $a$ in $P_{M}^{\prime}$ would have to be in $M$. So $a$ is in a stable $M$ if and only if there is a clause in $P^{\prime}$ whose single constraint is satisfied by $M$. Clearly, this is the same condition as for $a$ being in a supported model of $P^{\prime}$. Hence, the stable models of $P^{\prime}$ are the same as the supported models of $P^{\prime}$ and in turn the supported models of $P$. Consequently, $L P_{k} \leq_{s} L P_{k}^{s u p}$.

Now take $P$ in $L P_{k}$. Let $a_{1}, \ldots, a_{n}$ be the variables of $P$. Let $N$ be the number of clauses in $P$. The problem of simulating $P$ with an $L P_{k}^{\text {sup }}$ program can be viewed as the problem of rewriting $P$ 's clauses without premises. Given an arbitrary subset $M$ of the Herbrand Universe we need some way to simulate the derivation of variables when we evaluate the Horn program $P_{M}$. In evaluating $P_{M}$, we need at most $n$ rounds before we reach a fixed point. To perform our simulation we introduce new variables $r_{i, t}$ for $1 \leq i \leq N$ and for $1 \leq t \leq N$. $r_{i, t}$ is supposed to keep track of whether rule $i$ of $P$ has fired by time $t$. Let $h$ be the function which takes clause numbers and returns the number of the variable which is at the head of the clause. Let $P^{\prime}$ be the following $L P_{k}^{s u p}$ program:

For each clause in $P$ of the form:

$$
a_{h(k)} \leftarrow: B
$$

We add to $P^{\prime}$ the clauses:

(1) $r_{h(k), t} \leftarrow: B$ where $1 \leq t \leq n$.

Next, for each clause in $P$ of the form:

$$
a_{h(k)} \leftarrow a_{h\left(i_{1}\right)}, \ldots, a_{h\left(i_{m}\right)}: B
$$

We add to $P^{\prime}$ the clause:

(2) $r_{h(k), t+1} \leftarrow: B \wedge\left(\mathbb{V}_{h(j)=h\left(i_{1}\right)} r_{j, t}\right) \wedge \ldots \wedge\left(\mathbb{W}_{h(j)=h\left(i_{m}\right)} r_{j, t}\right)$ where $1 \leq t \leq n-1$. Finally, we add to $P^{\prime}$ the clauses: 
(3) $a_{h(i)} \leftarrow: r_{i, n} \quad$ for $1 \leq i \leq N$

Suppose $M^{\prime}$ is a model of $P^{\prime}$ (since the clauses of $P^{\prime}$ have no premises, supported and stable models of $P^{\prime}$ will be the same). Let $M$ be $M^{\prime}$ restricted to the variables of $P$. Clauses in the Horn program $P_{M}$ inherit a numbering from the clauses they came from in $P$. Hence, clauses (1) and (2) of $P^{\prime}$ will guarantee that $r_{i, t}$ is valid in $M^{\prime}$ if and only if the $i$-th rule of $P_{M}$ could fire at time $t$ in the evaluation of $P_{M}$. So if $r_{i, n}$ is true then $a_{h(i)}$ could be derived in $P_{M}$. Thus, rule (3) assures us that a variable is in $M$ if and only if it could be derived in evaluating $P_{M}$. So $M$ will be a model of the Horn program $P_{M}$ and hence a stable model of $P$.

Suppose $M$ is a model $P$. We can extend this to a model of $P^{\prime}$ by assigning $r_{i t}$ true if rule $i$ has fired in $P_{M}$ by time $t$, and by assigning $r_{i t}$ false, otherwise. This completes the argument.

As before, let $D L_{k}$ with respect to supported models be denoted $D L_{k}^{\text {sup }}$, and $D L_{k}$ with respect to stable models we will just denoted by $D L_{k}$. One can easily modify the argument given above to porove the following.

Theorem 8. $D L_{k}^{\text {sup }} \leq_{s} D L_{k}$ and $D L_{k} \leq_{w s} D L_{k}^{\text {sup }}$. Thus, $D L_{k} \equiv_{w s} D L_{k}^{\text {sup }}$.

We will show below that $C C_{k} \underline{L}_{s} L P_{k}$. We know $L P_{k}^{\text {sup }} \mathbb{L}_{s} C C_{k}$ since models of $C C_{k}$ programs are pairwise incomparable as sets, yet supported models of an $L P_{k}$ program can be subsets of each other. However, if we slightly modify the definition of $L P_{k}^{s u p}$ to only consider programs whose supported models are pairwise disjoint then $C C_{k}$ is as representationally succinct as the resulting theory. Call this new theory $L P_{k}^{*}$. We can convert any $L P_{k}^{s u p}$ program $P$ over the variables the $p_{1}, \ldots p_{n}$ into an $L P_{k}^{*}$ program $P^{\prime}$ over the variables $p_{1}, \ldots, p_{n}, p_{1}^{\prime}, \ldots, p_{n}^{\prime}$. To make $P^{\prime}$ we add to $P$ the clauses $p_{i} \leftarrow: \neg p_{i}^{\prime}$ and $p_{i}^{\prime} \leftarrow: \neg p_{i}$ where $1 \leq i \leq n$. It is easy to see that $P^{\prime}$ has the same number of models as $P$ and every model in $P$ can be extended to a model of $P^{\prime}$. Further, each of $P^{\prime}$ 's models are pairwise incomparable. Thus, $L P_{k}^{*}$ is a natural modification of $L P_{k}^{s u p}$ since this construction shows $L P_{k}^{s u p} \equiv_{w s} L P_{k}^{*}$. Hence, by Theorem 7 it also shows that $L P_{k}^{*} \equiv{ }_{w s} L P_{k}$.

\section{Theorem 9.}

1. $L P_{k}^{*} \leq_{s} C C_{k}$ but $C C_{k} \not_{s} L P_{k}$ unless $\Sigma_{k+1}^{p} \subseteq \Delta_{k+1}^{p} /$ poly.

2. If $K$ is a reasoning formalism for which model checking can be done in $\Delta_{k+1}^{p}$ then $C C_{k} \mathbb{L}_{s} K$ unless $\Sigma_{k+1}^{p} \subseteq \Delta_{k+1}^{p} /$ poly.

Proof. To see $L P_{k}^{*} \leq_{s} C C_{k}$ let $P \in L P_{k}$. By conjoining constraint formulas we can assume that the clauses in $P$ are of the form:

$$
a_{i} \leftarrow p_{i 1}, \ldots p_{i n}: B_{i}
$$

Consider the circumscribed program $P^{\prime}$ made up of clauses of the form:

$$
a_{i} \leftarrow: \mathbb{A}_{j} p_{i j} \wedge B_{i}
$$


Suppose $M$ is a supported model of $P$. For each tail of a clause that $M$ satisfies in $P, M$ will satisfy the corresponding tail in $P^{\prime}$. Now if $M$ satisfies the tail of a clause $P$ it must also satisfy the head. $P_{M}^{\prime}$ is the conjunction of these heads and hence will be satisfied. As models for $P$ are pairwise disjoint there is no $m \subset M$ such that $m$ is a model of $P_{m}$. Hence, there is no $m$ such $m$ satisfies $\wedge P_{m}^{\prime}$. On the other hand, suppose $M^{\prime}$ is a model of $P^{\prime}$. If the tail of a clause in $P^{\prime}$ is satisfied by $M^{\prime}$, the head must also be satisfied since $M^{\prime}$ satisfies $P_{M^{\prime}}$. Thus, the corresponding clause in $P$ will also be satisfied. If tail of a clause is false in $P^{\prime}$ its corresponding clause will be false in $P$ as well. Thus, $M^{\prime}$ is a model of $P$. As $P$ was an arbitrary program in $L P_{k}^{*}$, we have shown $L P_{k}^{*} \leq_{s} C C_{k}$.

To see $C C_{k} \mathbb{Z}_{s} L P_{k}$ it suffices to show the second statement. That is, $C C_{k} \mathbb{Z}_{s}$ $K$ for any reasoning formalism for which model checking can be done in $\Delta_{k+1}^{p}$. Suppose $F=\exists \boldsymbol{x} E(\boldsymbol{x})$ is a $\Sigma_{k+1}^{q}$-sentence. Then we can convert $F$ to a prenex normal form $\Sigma_{k+1}^{q}$-sentence whose matrix is in 3CNF. Our first step in this proof is to come up with a single formula which will allow us to code up sentences of this type. Our formula will use the variables $x_{01}, \ldots, x_{0 n}, \ldots, x_{k 1}, \ldots, x_{k n}$. There are $8 \cdot\left(\begin{array}{c}(k+1) n \\ 3\end{array}\right)$ possible $3 \mathrm{CNF}$ clauses over these variables. For each possibility our formula will have a variable $c_{i}$. We will denote the literals in clause $i$ by $l_{i 1}, l_{i 2}, l_{i 3}$. We are now ready to state our formula.

Let $G_{n}\left(\boldsymbol{c}, \boldsymbol{x}_{\mathbf{0}}\right)$ be the formula:

$$
\forall x_{11} \ldots x_{1 n} \exists x_{21} \ldots x_{2 n} \ldots Q_{k 1} \ldots x_{k n}\left(\mathbb{X}_{1 \leq i \leq 8 \cdot\left(\begin{array}{c}
(k+1) n \\
3
\end{array}\right)} c_{i} \supset l_{i 1} \vee l_{i 2} \vee l_{i 3}\right)
$$

Given $\Sigma_{k+1}^{q}$-formula $F$, let $n$ be the number of variables in $F$. By choosing the values of the $c_{i}$ 's appropriately we can encode $F$ as $F^{\prime}=\exists \boldsymbol{x}_{\mathbf{0}} G_{n}\left(\boldsymbol{c}, \boldsymbol{x}_{\mathbf{0}}\right)$. This encoding is polynomial in the size of $F$.

Let $P[n]$ be the following circumscribed program:

$$
\begin{aligned}
& \mathbb{X}_{i} c_{i} \not \equiv c_{i}^{\prime} \leftarrow: \\
& x_{0 i} \leftarrow: y \\
& y \leftarrow: \neg G_{n}\left(\boldsymbol{c}, \boldsymbol{x}_{\mathbf{0}}\right) .
\end{aligned}
$$

Let $m_{F}$ be the set that contains $y$, all the $x_{0 i}$ 's, and $c_{i}$ if $l_{i 1} \vee l_{i 2} \vee l_{i 3}$ is in the matrix of $F$, and $c_{i}^{\prime}$ otherwise. We claim $F$ is satisfiable if and only if $m_{F}$ is not a model of the program $P[n]$. Indeed, if $F$ is modeled by $M$ (i.e. satisfiable), then extend $M$ by adding those $c_{i}$ 's and $c_{i}^{\prime}$ 's which are in $m_{F}$. Since $\nu_{M}\left(G_{n}\left(\boldsymbol{c}, \boldsymbol{x}_{\mathbf{0}}\right)\right)=1, P[n]_{M}$ will be just $\mathbb{X}_{i} c_{i} \not \equiv c_{i}^{\prime}$ and $M$ models this formula. $M$ does not contain $y$ and is contained in $m_{F}$. So $m_{F}$ is not a model of $P[n]$.

To prove converse, assume that $m_{F}$ is not a model of $P[n]$. Then there must be an $M \subset m_{F}$ such that $\nu_{M}\left(\mathbb{X} P[n]_{M}\right)=1$. By (1) in $P[n], M$ and $m_{F}$ must each contain the same $c_{i}$ 's and $c_{i}^{\prime}$. $M$ must not contain $y$ otherwise by (2) we would have $M=m_{F}$. Thus, there must be some choice of $\boldsymbol{x}_{\mathbf{0}}$ which satisfied $G_{n}$. This would in turn mean that $F$ is satisfiable.

Suppose now that $C C_{k} \leq_{s} K$ where model checking in $K$ is in $\Delta_{k+1}^{p}$. Then for each $n$ there is an at most polynomially larger program $P^{\prime}[n]$ in $K$ with the same models as $P[n]$. The program $P^{\prime}[n]$ will be our polynomial sized advice. We can now check if a given $n$ variable $\Pi_{k+1}^{q}$ formula $\neg F$ is valid: we check if $m_{F}$ 
is a model of $P^{\prime}[n]$. This can be done in $\Delta_{k+1}^{p}$. Thus, $\Pi_{k+1}^{p} \subseteq \Delta_{k+1}^{p} /$ poly which in turn implies $\Sigma_{k+1}^{p} \subseteq \Delta_{k+1}^{p} /$ poly as $\Delta_{k+1}^{p} /$ poly is closed under complement.

We notice that both the Theorem 9 and its proof generalize one of the main results of [8].

Corollary 3. $C C_{k}$ 志ws $L P_{k}$ unless $\Pi_{k+1}^{p} \subseteq \Sigma_{k+1}^{p} /$ poly.

Proof. Let $P[n]$ be as above. Suppose $C C_{k} \leq_{w s} L P_{k}$. Let $P^{\prime}[n]$ be the $L P_{k}$ program corresponding to $P[n]$. To check if a given $n$ variable $\Pi_{k+1}^{q}$ formula $\neg F$ is valid, we check if $m_{F}$ can be expanded to a model of $P^{\prime}[n]$. This can be done in $\Sigma_{k+1}^{p}$. Thus, $\Pi_{k+1}^{p} \subseteq \Sigma_{k+1}^{p} /$ poly.

Theorem 10. $C C_{k} \leq_{s} D L_{k}$ but $D L_{k} \mathbb{E}_{s} C C_{k}$ unless $\Sigma_{k+1}^{p} \subseteq \Pi_{k+1}^{p} /$ poly.

Proof. To see $C C_{k} \leq_{s} D L_{k}$ let $P \in C C_{k}$. Suppose $P$ has free variables $p_{1}, \ldots p_{m}$ and it is made up of clauses of the form $A_{i} \leftarrow: B_{i}$ where $1 \leq i \leq n$. Let $V(\boldsymbol{p})$ be the formula $\mathbb{M}_{i} B_{i} \supset A_{i}$. Let $W$ be empty and let $D$ be

$$
\left\{d_{j}=\frac{: \neg p_{j}, V(\boldsymbol{p})}{\neg p_{j}} \mid 1 \leq j \leq m .\right\}
$$

Suppose $M$ is model of $P$. $M$ must satisfy $V(\boldsymbol{p})$. We can obtain an extension of $\langle D, W\rangle$ by applying those $d_{j}$ 's for which the $p_{j} \notin M$. Since $M$ is minimal no more defaults will fire. The only model of this extension is $M$. On the other hand, if $S$ is an extension of $\langle D, W\rangle$ then its only model is the one that sets the $p_{j}$ 's to zero only if $d_{j}$ fired. Further, this model is a minimal model of the formula $V(\boldsymbol{p})$ and hence a minimal model of $P$.

To see $D L_{k} \not \mathbb{s}_{s} C C_{k}$ unless $\Sigma_{k+1}^{p} \subseteq \Pi_{k+1}^{p} /$ poly, we consider a special type of $\Sigma_{k+1}^{q}$-sentence. We call a $\Sigma_{k+1}^{q}$-formula pure if it is in prenex normal form, its matrix is in $3 \mathrm{CNF}$ and, for each clause, either all the literals are positive or all the literals are negative. Satisfiability for a pure propositional formulas is $N P$-complete [7]. It follows that deciding whether a given pure $\Sigma_{k+1}^{q}$-sentence is valid is $\Sigma_{k+1}^{p}$-complete.

We are now ready to define the default theory we will need in our succinctness result. We will use the following formula $P\left(\boldsymbol{X}, \boldsymbol{Y}, \boldsymbol{c}, \boldsymbol{c}^{\prime}\right)$ to allow us to code up pure $\Sigma_{k+1}^{q}$-sentences involving $n$ variables:

$$
\begin{gathered}
\forall x_{11} \ldots x_{1 n} \exists x_{21} \ldots x_{2 n} \ldots Q_{k 1} \ldots x_{k n}\left[\mathbb{A}_{1 \leq i \leq\left(\begin{array}{c}
(k+1) n \\
3
\end{array}\right)}\left(c_{i} \supset l_{i_{1}} \vee l_{i_{2}} \vee l_{i_{3}}\right)\right. \\
\left.\wedge \mathbb{A}_{1 \leq i \leq\left(\begin{array}{c}
(k+1) n \\
3
\end{array}\right)}\left(c_{i}^{\prime} \supset l_{i_{1}}^{\prime} \vee l_{i_{2}}^{\prime} \vee l_{i_{3}}^{\prime}\right)\right]
\end{gathered}
$$

A literal $l_{j_{v}}$ is either a variable $x_{i t}$ or a formula $X_{i}$. A literal $l_{j_{v}}^{\prime}$ is either a literal of the form $\neg x_{i t}$ or a formula $Y_{i}$. The three literals in the disjuncts are supposed to run over all possible ways one could pick $l_{j_{1}}, l_{j_{2}}, l_{j_{3}}$ or $l_{j_{1}}^{\prime}, l_{j_{2}}^{\prime}, l_{j_{3}}^{\prime}$ from their corresponding set of variables. The variables $c_{i}$ and $c_{i}^{\prime}$ are supposed to control if a given disjunct of three literals is turned on. We define the formulas $X_{i}$ and $Y_{i}$ below. $X_{i}$ is the formula:

$$
\neg b \vee \neg z_{1} \vee \cdots \vee \neg z_{i-1} \vee z_{i} \vee \neg z_{i+1} \vee \cdots \vee \neg z_{n}
$$


$Y_{i}$ is the formula:

$$
b \vee \neg z_{1} \vee \cdots \vee \neg z_{i-1} \vee z_{i} \vee \neg z_{i+1} \vee \cdots \vee \neg z_{n}
$$

Let $\Delta[n]=\langle D, W\rangle$ be the default theory with $W$ defined by:

$$
W=W_{i=1}^{n} \neg X_{i} \vee W_{i=1}^{n} \neg Y_{i} \vee\left(b \wedge \mathbb{A}_{i=1}^{n} z_{i}\right)
$$

and $D$ consisting of the following defaults:

$$
\begin{array}{lll}
\text { (1) } \frac{: P\left(\boldsymbol{X}, \boldsymbol{Y}, \boldsymbol{c}, \boldsymbol{c}^{\prime}\right) \wedge c_{i}}{c_{i}} & \frac{: P\left(\boldsymbol{X}, \boldsymbol{Y}, \boldsymbol{c}, \boldsymbol{c}^{\prime}\right) \wedge c_{i}^{\prime}}{c_{i}^{\prime}} & \text { for } \quad 1 \leq i \leq\left(\begin{array}{l}
n \\
3
\end{array}\right) \\
\text { (2) } \frac{: \neg c_{i}}{\neg c_{i}} & \frac{: \neg c_{i}^{\prime}}{\neg c_{i}^{\prime}} & \text { for } \quad 1 \leq i \leq\left(\begin{array}{l}
n \\
3
\end{array}\right) \\
\text { (3) } \frac{: \neg X_{i}}{Y_{i}} & \frac{: \neg Y_{i}}{X_{i}} & \text { for } \quad 1 \leq i \leq n .
\end{array}
$$

Notice that $\langle D, W\rangle$ is polynomial (in fact cubic) in $n$.

Let $F=\exists \boldsymbol{x}_{\mathbf{0}} E\left(\boldsymbol{x}_{\mathbf{0}}\right)$ be a pure $\Sigma_{k+1}^{q}$-sentence over $n$ variables. We can view $F$ as being over some subset of the variables in $x_{i j}$ where $0 \leq i \leq k$ and $1 \leq j \leq n$. To each clause in its matrix we can find a corresponding clause in $P\left(\boldsymbol{X}, \boldsymbol{Y}, \boldsymbol{c}, \boldsymbol{c}^{\prime}\right)$. To find the corresponding clause to a clause in $F$ containing a literal $x_{0 j}$ or $\neg x_{0 j}$ we view $x_{0 j}$ as $Y_{j}$ and $\neg x_{0 j}$ as $X_{j}$. Let $m_{F}$ be the subset of the Herbrand base of $\Delta[n]$ which contains $b$ and $z_{1} \ldots z_{n}$, and contains $c_{i}, c_{i}^{\prime}$ if the corresponding clause appears in $F$. We claim $m_{F}$ is a model of an extension of $\Delta[n]$ if and only if $F$ is valid. Suppose $F$ is valid and let $x_{0 i_{1}} \ldots x_{0 i_{m}}$ be a vector which satisfies $E$. Let $Z$ contain $c_{i}$ if $c_{i} \in m_{F}$ and $\neg c_{i}$ if $c_{i} \notin m_{F}$. Likewise, let $Z$ contain $c_{i}^{\prime}$ if $c_{i}^{\prime} \in m_{F}$ and $\neg c_{i}^{\prime}$ if $c_{i}^{\prime} \notin m_{F}$. For each $1 \leq i \leq n$ let $Z$ contain $Y_{i}$ if $i$ is an 'on' bit in the assignment satisfying $E$ and if not let $Z$ contain $X_{i}$. $C n(Z \cup W)$ will be a an extension. Since $F$ was valid, $P\left(\boldsymbol{X}, \boldsymbol{Y}, \boldsymbol{c}, \boldsymbol{c}^{\prime}\right)$ is consistent with $W$ and $Z$. Thus, rules (1) and (2) will let us derive back precisely the $c_{i}, c_{i}^{\prime}, \neg c_{j}$, and $\neg c_{j}^{\prime}$ 's in $Z$. Next, the rule (3) forces us to derive back the same $X_{i}$ 's and $Y_{i}$ 's. It is easy to see that $m_{F}$ is a model of $C n(Z \cup W)$. On the other hand, if $m_{F}$ is a model of $\Delta[n]$, we need to show $F$ is valid. Let $S$ be the extension that $m_{F}$ models. $S$ must have exactly those $c_{i}$ 's and $c_{i}^{\prime}$ 's needed to code $F$ into $P$. This follows from the definition of $m_{F}$ and the fact that $m_{F}$ models $S$. Now in order for $D_{S}$ to be able to derive back these $c_{i}$ 's and $c_{i}^{\prime}$ 's there must be some choice of the $X_{i}$ 's and $Y_{i}$ 's such that $P$ is consistent with $S$. The rules (3) imply that only one of $X_{i}$ and $Y_{i}$ can hold for each $i$. We can thus assign $x_{0 i}$ true if $Y_{i}$ holds and $x_{0 i}$ false otherwise. Since $P$ was consistent with $S$ and as $F$ was encoded with the $c_{i}$ 's and $c_{i}^{\prime}$ into $P$ this assignment must satisfy $E$. Hence, $F$ is valid as $E$ satisfiable.

Suppose $D L_{k} \leq_{s} C C_{k}$. Then for each $n$ there is circumscribed program $D[n]$ with the same models as the theory $\Delta[n]$ and polynomial in the size of $\Delta[n]$. These $D[n]$ will be our polynomial sized advice. To decide if a pure $\Sigma_{k+1}^{q}$-sentence on $n$ variables is valid one need only check if $m_{F}$ is a model of $D[n]$. This involves checking that no subset $M$ of $m_{F}$ satisfies $\mathbb{A} D[n]_{M}$, which can be done in $\Pi_{k+1}^{p}$. Thus, we reduced a $\Sigma_{k+1}^{p}$ problem to a $\Pi_{k+1}^{p} /$ poly one. 
Theorem 11. $D L_{k} \leq_{w s} L P_{k+1}$.

Proof. Consider a $D L_{k}$ theory $\langle D, W\rangle$ using variables $x_{1}, \ldots, x_{n}$. By Theorem 8 it suffices to consider the case where defaults in $\langle D, W\rangle$ are prerequisite-free. Our first task is to come up with a formula $\operatorname{Model}_{\langle D, W\rangle}(\boldsymbol{x})$ which will be true if and only if a truth assignment to $\boldsymbol{x}$ is a model of $\langle D, W\rangle$. By conjoining constraint formulas together we can assume defaults in $\langle D, W\rangle$ to be of the form:

$$
\frac{: C_{i}(\boldsymbol{x})}{\gamma_{i}(\boldsymbol{x})} .
$$

Let $Z W(\boldsymbol{x}, \boldsymbol{y})$ be the following formula:

$$
\mathbb{A}_{i}\left(\gamma_{i}(\boldsymbol{x}) \supset\left(W(\boldsymbol{y}) \wedge \gamma_{i}(\boldsymbol{y})\right) .\right.
$$

$Z W$ will play the role that $Z \cup W$ plays in extension existence. In extension existence, however, we need to guess a subset of the conclusion of rules. In the present case, we will be given a model, so it will be only necessary to see which conclusions the model satisfies. We now introduce a formula which expresses that the $i$ th rule of $D$ contributes to $D_{S}$. Let $D_{S, i}(\boldsymbol{x})$ be the formula:

$$
\forall \boldsymbol{y} C_{i}(\boldsymbol{y}) \wedge Z W(\boldsymbol{y}, \boldsymbol{x}) .
$$

Using $Z W$ and $D_{S, i}$ we can now express $\operatorname{Model}_{\langle D, W\rangle}(\boldsymbol{x})$ as the formula:

$$
\left.Z W(\boldsymbol{x}, \boldsymbol{x}) \wedge \forall \boldsymbol{z}\left[\left(D_{S, i}(\boldsymbol{x}) \supset \gamma_{i}\right)\right) \equiv Z W(\boldsymbol{z}, \boldsymbol{x})\right]
$$

This is a $Q B F_{k+1}$ formula so it can be used in $L P_{k+1}$ programs. The following short $L P_{k+1}$ program $P$ has the same models as $\langle D, W\rangle$ :

$$
\begin{aligned}
& x_{i} \leftarrow: \operatorname{Model}_{\langle D, W\rangle}\left(x_{1}, \ldots, x_{n}\right) \wedge x_{i} \quad i=1, \ldots, n \\
& x_{1} \leftarrow:\left(\neg \operatorname{Model}_{\langle D, W\rangle}\left(x_{1}, \ldots, x_{n}\right)\right) \wedge \mathbb{N}_{1 \leq i \leq n}\left(\neg x_{i}\right) .
\end{aligned}
$$

Let $\nu$ be a truth assignment. Suppose $\nu$ is model of $\langle D, W\rangle$. Then clauses (1) permit derivations of all those $x_{i}$ 's for which $\nu\left(x_{i}\right)=1$. Moreover, no other $x_{i}$ 's can be derived. On the other hand, if we have a truth assignment $\nu$ to the $x_{i}$ 's which is not a model of $\langle D, W\rangle$ then there are two cases. Either none of the $x_{i}$ 's with $\nu\left(x_{i}\right)=1$ will be derivable from $P$ or $\nu$ has all the $x_{i}$ 's set to zero in which case $x_{1}$ can be derived from $P$. In either case, $\nu$ is not a model of $P$.

Thus, the models of $P$ are precisely the models of $\langle D, W\rangle$. Notice that the clauses in $P$ have no premises so stable and supported models of $P$ will coincide. So we have shown both $D L_{k} \leq_{w s} L P_{k+1}$ and $D L_{k} \leq_{w s} L P_{k+1}^{\text {sup }}$.

Let $\operatorname{Model}_{\phi_{K}}(\boldsymbol{x})$ be the predicate which is true if and only if $\boldsymbol{x}$ is a model of the knowledge base $\phi_{K}$ in the reasoning formalism $K$. Since satisfaction of a $Q B F_{k}$ formula is a $\Sigma_{k+1}^{p}$-complete problem, it seems likely that we can express Model $_{\phi_{K}}(\boldsymbol{x})$ as a $Q B F_{k}$ formulas for many formalisms for which model checking is in $\Delta_{k+1}^{p}$. Then using essentially the same program as in Theorem 11 we can show: 
Corollary 4. Suppose $K$ is a propositional reasoning formalism for which the

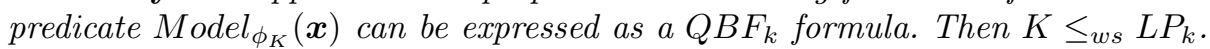

This gives some evidence that $L P_{k}, C C_{k}$, and $D L_{k}$ are nice hierarchies in which to study the succinctness of reasoning formalisms for which model checking is in the polynomial hierarchy.

Theorem 12. $L P_{k} \leq_{s} D L_{k}$ but $D L_{k} \not_{s} L P_{k}$ unless $\Sigma_{k+1}^{p} \subseteq \Pi_{k+1}^{p} /$ poly.

Proof. The second part of the theorem follows from Theorem 9 and Theorem 10. To prove the first part, let $P \in L P_{k}$. We translate $P$ into the default theory $\langle D, \emptyset\rangle$ where clauses of the form

$$
p \leftarrow a_{1}, \ldots, a_{n}: B_{1}\left(\boldsymbol{b}_{1}\right), \ldots, B_{n}\left(\boldsymbol{b}_{m}\right)
$$

become defaults:

$$
\frac{a_{1} \ldots, a_{n}: B_{1}\left(\boldsymbol{b}_{1}\right), \ldots, B_{n}\left(\boldsymbol{b}_{m}\right)}{p} .
$$

It is easy to see $P$ and $\langle D, W\rangle$ have the same models.

Theorem 13. $L P_{k+1} \leq_{w s} D L_{k}$.

Proof. Given an $L P_{k+1}$ program $P$ with $Q B F_{k+1}$ constraints we first want to convert it to an $L P_{k+1}$ program with all constraint formulas $\Sigma_{k+1}^{q}$ or $\Pi_{k+1}^{q}$. By conjoining the constraint formulas we can assume each clause has only one constraint formula. By pushing negations inward we can assume each constraint formula involves only AND's and OR's of $\Sigma_{k+1}^{q}$ and $\Pi_{k+1}^{q}$ formulas. We eliminate propositional connectives one at a time for these $Q B F_{k+1}$ constraint formulas by repeatedly using one of the following transformations. A clause of the form:

$$
a \leftarrow p_{1}, \ldots, p_{n}: B \wedge C
$$

where $B \wedge C \notin \Sigma_{k+1}^{q}$ becomes

$$
\begin{aligned}
& b_{1} \leftarrow p_{1}, \ldots, p_{n}: B \wedge b_{2} \\
& b_{2} \leftarrow p_{1}, \ldots, p_{n}: b_{1} \wedge C \\
& a \leftarrow b_{1} \wedge b_{2}:
\end{aligned}
$$

where $b_{i}$ are new variables not appearing in $P$. Notice in any model of the resulting program either $a, b_{1}$ and $b_{2}$ are true or they are all false. Thus, so far the transformation is a weakly succinct one. A clause of the form:

$$
a \leftarrow p_{1}, \ldots, p_{n}: B \vee C
$$

where $B \vee C \notin \Sigma_{k+1}^{q}$ becomes

$$
\begin{aligned}
& a \leftarrow p_{1}, \ldots, p_{n}: B \\
& a \leftarrow p_{1}, \ldots, p_{n}: C .
\end{aligned}
$$


This type transformation adds no variables and does not change the models of $P$. Hence, applying both these types of transformations to clauses in $P$ will yield a program $P^{\prime}$ and the total transformation is a weakly succinct one. Once every constraint is either $\Sigma_{k+1}^{q}$ or $\Pi_{k+1}^{q}$, we convert the resulting logic program $P^{\prime}$ into a default theory $\langle D, W\rangle$ where $W=\emptyset$. Each clause in $P^{\prime}$ of the form:

$$
a \leftarrow p_{1}, \ldots, p_{n}: A
$$

is converted into a default of the form:

$$
\frac{\mathbb{X} p_{i}: A}{a}
$$

Given a model $M$ of $P^{\prime}$ it is obvious that $C n(M)$ will be an extension of $\langle D, W\rangle$, so $M$ will be a model of $\langle D, W\rangle$. Conversely, the variables which appear in an extension of $\langle D, W\rangle$ will give a model of $P^{\prime}$.

To make $\langle D, W\rangle$ a $D L_{k}$ theory we replace each $\Sigma_{k+1}^{q}$ constraint formula $A=\exists x_{1}, \ldots x_{n} B(\boldsymbol{x}, \boldsymbol{c})$ by a new variable $\gamma_{A}$. We then add defaults of the form:

$$
\frac{: b_{i}}{b_{i}} \quad \frac{: \neg b_{i}}{\neg b_{i}} \quad \text { for } 1 \leq i \leq n
$$

and

$$
\frac{: B(\boldsymbol{b}, \boldsymbol{c})}{\gamma_{A}}
$$

where $b_{i}$ are new variables. The defaults $(* *)$ guarantee any extension of the resulting theory will have some choice of the $b_{i}$ 's. Thus, $\gamma_{A}$ will be in any such extension if and only if $\exists \boldsymbol{b} B(\boldsymbol{b}, \boldsymbol{c})$.

We replace each $\Pi_{k+1}^{q}$ constraint formula $A=\forall x_{1}, \ldots, x_{n} B(\boldsymbol{x}, \boldsymbol{c})$ by a new variable $\gamma_{A}$ and add a default:

$$
\frac{: B(\boldsymbol{b}, \boldsymbol{c})}{\gamma_{A}}
$$

where $b_{i}$ are new variables. When we try to find an extension $S$ of the resulting default theory, $B(\boldsymbol{b}, \boldsymbol{c})$ will be consistent with $S$ if and only if $\forall \boldsymbol{b} B(\boldsymbol{b}, \boldsymbol{c})$ holds. So $\gamma_{A}$ will be if $S$ if and only if $\forall \boldsymbol{b} B(\boldsymbol{b}, \boldsymbol{c})$ holds.

Thus, extensions of $\langle D, W\rangle$ can be made extensions of $\left\langle D^{\prime}, W^{\prime}\right\rangle$ by adding appropriate choices of $\gamma_{A}$ 's. Eliminating such variables from an extension of $\left\langle D^{\prime}, W^{\prime}\right\rangle$ will yield an extension of $\langle D, W\rangle$.

Consequently, $\left\langle D^{\prime}, W^{\prime}\right\rangle$ is a weakly succinct transformation of $\langle D, W\rangle$ and so also of $P$. Thus, $L P_{k+1} \leq_{w s} D L_{k}$.

We get then the following corollary:

Corollary 5. $D L_{k} \equiv_{w s} L P_{k+1}$.

Using the results of Baker, Gill, Solovay [1] and Furst, Saxe, Sipser [6] we can construct an oracle $A$ for which $\Sigma_{k}^{p}(A) \neq \Pi_{k}^{p}(A)$ for $k \geq 1$. The various conditional succinctness inequalities we have obtained above thus become strict for 
the formalisms $L P_{k}^{*}(A), L P_{k}(A), C C_{k}(A)$ and $D L_{k}(A)$. Similarly, if we choose a $P S P A C E$-complete oracle then as the polynomial hierarchy collapses to the first level with respect to this oracle so to do our succinctness hierarchies. In the same vein, the translations used above show that $L P_{\infty} \equiv_{w s} C C_{\infty} \equiv_{w s} D L_{\infty}$.

We now spend a moment to mention the results of the interesting paper of Eiter, Lu, and Subrahmanian [5] which also appears in this volume. Their setting is predicate logic programming. They develop a notion of constrained interpretation and constrained logic program to avoid having to define the stable models of logic programs in terms of minimal Herbrand models of their ground instantiations which may, in general, be infinite. In the function free case their constraints turn out to be first-order formulas in the language with equality. For bounded numbers of quantifier alternations the problem of satisfaction or validity of such formulas is complete for various levels of the polynomial hierarchy and so in turn problems concerning constrained logic programs are complete for various levels of the hierarchy. It might be possible to interpret their results into a reasoning formalism with quantified Boolean constraints since if we have an equality $X=Y$ where $X, Y$ can range over finitely many values we can do a propositional translation of this equality as $\wedge_{i}\left(x_{i} \Leftrightarrow y_{i}\right)$ where $x_{i}$ and $y_{i}$ represent the bits in the coding of $X$ and $Y$. Such translations might be of practical importance in a hardware implementation of their scheme and warrant further study.

\section{References}

1. T. Baker, J. Gill, and R. Solovay ."Relativizations of the $\mathrm{P}=$ ? NP question". SIAM Journal of Computing. , 1993. 431-442.

2. M. Cadoli and M. Schaerf. 'A survey on complexity results for non-monotonic logic" Journal of Logic Progrmming. , Nov. 1993, vol.17,(no.2-4):127-60.

3. W.F. Dowling and J.H. Gallier. "Linear-time algorithms for testing the satisfiability of propositional Horn formulae". Journal of Logic Programming, 3:267-284, 1984

4. T. Eiter and G. Gottlob. "Propositional circumscription and extended closed-world reasoning are $\Pi_{2}$-complete". Theoretical Computer Science, 114:231-245, 1993.

5. T. Eiter and J. Lu and V.S.Subrahmanian. "Computing non-ground representations of stable models". In: Logic Programming and Nonmonotonic Reasoning, LPNMR'97 This volume

6. M. Furst and J.B.Saxe and M. Sipser. "Parity, circuits and the polynomial hierarchy". Math Systems Theory, 17:13-27, 1984.

7. M. R. Garey and D. S. Johnson. Computers and Intractibility: A Guide to the theory of NP-completeness. W.H. Freeman and Company, 1979

8. G. Gogic , H. Kautz, C. Papadimitriou, and B. Selman. "The comparative linguistics of knowledge representation". In Proceedings of International Joint Conference on Artificial Intelligence, IJCAI-95. Springer Lecture notes in Artificial Intelligence; 1042. pages 862-869, 1995.

9. G. Gogic. Complexity Aspects of Knowledge Representation. Ph.D. Thesis. Computer Science Department, U.C. San Diego. 1996.

10. J. Goldsmith and D. Joseph. Three results on the polynomial isomorphism of complete sets. In Foundations of Computer Science, volume 27, 1986. 
11. G. Gottlob. "Complexity results for nonmonotonic logics". Journal of Logic and Computation, 2:397-425, 1992

12. J. Jaffar and J.-L. Lassez. Constraint Logic Programming. In: Proceedings of the 14th ACM Symposium on Principles of Programming Languages, pages 111-119, Münich, 1987.

13. J. Jaffar and M. Maher Constraint Logic Programming: A Survey. Journal of Logic Programming 19-20, pages 503-581. 1994.

14. V.W. Marek and M. Truszczyński. Nonmonotonic Logic. Springer Verlag. 1993.

15. V.W. Marek, A. Nerode and J.B. Remmel. "On logical constraints in Logic Programming". In: Logic Programming and Nonmonotonic Reasoning, LPNMR'95, Springer Lecture Notes in Artificial Intelligence 928, pages 43-56, 1995.

16. A.R. Meyer and L.J. Stockmeyer. "The equivalence problem for regular expressions with squaring requires exponential time". In Proceedings of the 13th annual symposium on switching and automata theory, pages 125-129. New York, NY: IEEE Computer Society, 1972.

17. C.H. Papadimitriou. "The complexity of knowledge representation". In Proceedings of the 11th Annual IEEE Conference on Computational Complexity, pages 244-248. IEEE Computer Society, 1996.

18. U. Schöning. "A note on complete sets for the polynomial hierarchy". $A C M$ SIGACT News 13 pages 30-34. 1981 